\title{
Posterior cortical atrophy and Alzheimer's disease: a meta-analytic review of neuropsychological and brain morphometry studies
}

\author{
Jorge Alves • José Miguel Soares • Adriana Sampaio • \\ Óscar F. Gonçalves
}

(C) Springer Science+Business Media New York 2013

\begin{abstract}
This paper presents the first systematic review and meta-analysis of neuropsychological and brain morphometry studies comparing posterior cortical atrophy (PCA) to typical Alzheimer's disease (tAD). Literature searches were conducted for brain morphometry and neuropsychological studies including a PCA and a tAD group. Compared to healthy controls (HC), PCA patients exhibited significant decreases in temporal, occipital and parietal gray matter (GM) volumes, whereas tAD patients showed extensive left temporal atrophy. Compared to tAD patients, participants with PCA showed greater GM volume reduction in the right occipital gyrus extending to the posterior lobule. In addition, PCA patients showed less GM volume loss in the left parahippocampal gyrus and left hippocampus than tAD patients. PCA patients exhibit significantly greater impairment in Immediate Visuospatial Memory as well as Visuoperceptual and Visuospatial Abilities than patients
\end{abstract}

Electronic supplementary material The online version of this article (doi:10.1007/s11682-013-9236-1) contains supplementary material, which is available to authorized users.

J. Alves · A. Sampaio · Ó. F. Gonçalves

Neuropsychophysiology Lab, CIPsi, School of Psychology,

University of Minho, 4710-057 Braga, Portugal

J. M. Soares

Life and Health Sciences Research Institute (ICVS), School of Health Sciences, University of Minho, Campus Gualtar, 4710-057 Braga, Portugal

J. M. Soares

ICVS/3B's - PT Government Associate Laboratory,

Braga/Guimarães, Portugal

J. Alves $(\bowtie)$

Laboratório de Neuropsicofisiologia, Escola de Psicologia,

Universidade do Minho, Campus de Gualtar,

4710-057 Braga, Portugal

e-mail: jorge.alves@psi.uminho.pt with tAD. However, tAD patients showed greater impairment in Delayed Auditory/Verbal Memory than patients with PCA. PCA is characterized by significant atrophy of the occipital and parietal regions and severe impairments in visuospatial functioning.

Keywords Alzheimer's disease (AD) · Posterior cortical atrophy $\cdot$ Magnetic resonance imaging (MRI) $\cdot$ Voxel-based morphometry $(\mathrm{VBM}) \cdot$ Meta-analysis $\cdot$ Effect Size Signed Differential Mapping (ES-SDM)

\section{Introduction}

The hallmark of typical Alzheimer's disease (tAD) is the onset of a specific pattern of episodic memory impairment (Clare et al. 2003) that is associated with abnormalities in the entorhinal cortex and hippocampus (Backman et al. 2004; Braak and Braak 1991; Scahill et al. 2002; Yamasaki et al. 2012).

However, several studies have documented impairments in other cognitive domains early in the progression of Alzheimer's disease, including executive function, controlled inhibitory processes, verbal abilities, visuospatial skills, attention and perceptual speed (Amieva et al. 2004; Backman et al. 2004; Yamasaki et al. 2012).

Though the early stages of Alzheimer's dementia are traditionally characterized in terms of memory deficits, nonamnestic presentations have also been considered in diagnostic criteria such as those proposed by the National Institute on Aging and the Alzheimer's Association (McKhann et al. 2011). Although brain pathology similar to that in TAD is found in atypical, nonamnestic variants of Alzheimer's disease, unusual cognitive profiles have been clinically observed. These variants have been consistently described as affecting predominantly language or visuospatial skills (Galton et al. 2000). 
One of the most frequently referred Alzheimer's disease variants is posterior cortical atrophy (PCA) (Benson et al. 1988). Neuroimaging in patients with PCA commonly shows hypoperfusion, hypometabolism and/or atrophy of occipital and parietal regions (Nestor et al. 2003). These presentations may result in visuospatial symptoms, such as apraxia, alexia and spatial neglect (see Kas et al. 2011, for PCA diagnostic criteria based on McMonagle et al. 2006). PCA is somewhat rare, as epidemiological estimates show that PCA accounts for approximately $4 \%$ of all new dementia cases observed in a memory clinic (Croisile 2004). Pathology studies report that most cases of PCA, upon autopsy, show Alzheimer's disease neuropathology (Victoroff et al. 1994) with similar amyloid-b burden (de Souza et al. 2011). As noted by Croisile (2004), Alzheimer's disease is characterized by underlying neuritic plaques and neurofibrillary tangles, which can be present anywhere in the brain and result in a diverse array of symptoms. Using this rationale, PCA may be considered a visual variant of Alzheimer's disease. Similarly, differences in the distribution of amyloid deposits, neurofibrillary tangles and neuronal loss may be responsible for the various clinical presentations observed in Alzheimer's disease.

Recent advances in neuroimaging methods and neuropsychological assessment techniques have led to improved clinical descriptions of Alzheimer's disease and increased knowledge of its various presentations. For example, studies of cortical density and thickness that directly compare PCA and $\mathrm{ADD}$ have reported significantly more atrophy of the visual association areas (e.g., the right parietal, bilateral posterior parietal regions and the occipital lobe) in PCA. Additionally, tAD patients were found to have greater volume decreases in the hippocampi and the left medial temporal lobe (Feldmann et al. 2008; Lehmann et al. 2011; Whitwell et al. 2007). Consistent with these findings, several functional imaging studies provide evidence for decreased activation in visual attention areas and the occipital lobes, as well as increased hypometabolism in the inferior occipitotemporal cortex in PCA (Feldmann et al. 2008; Kas et al. 2011). Similarly, when compared to tAD patients in structural imaging studies, PCA patients were found to have a greater global amyloid load in occipital and parietal regions as well as reduced white matter (WM) integrity in posterior regions and severe WM occipital atrophy (Crutch et al. 2012; Formaglio et al. 2011; Migliaccio et al. 2012a, b).

While these findings are intriguing, the results of individual studies are inherently limited and may lack sufficient statistical power due to small sample sizes, the inclusion of heterogeneous subjects, or the use of different clinical methods to make diagnostic classifications of the participants, which leads to discrepancies in describing the etiology of the disorder. To address these issues, tAD and PCA must be compared by a systematic review and meta-analysis of the neuropsychological deficits and brain morphometry findings reported in the literature.

Because previous studies report that patterns of brain atrophy (e.g., medial temporal lobe, as shown by Westman et al. 2011) are highly sensitive and specific in diagnosing Alzheimer's disease, we focused our study in comparing brain morphometry patterns in $\mathrm{ADD}$ and PCA, as well as the neuropsychological profiles associated with these conditions.

Voxel-based morphometry (VBM) is an automated whole-brain processing method, which allows the composition of brain tissue to be compared among (between and/or within) groups (Ashburner and Friston 2000). It has been used successfully in studies that examine changes in white and gray matter (GM) that occur in aging and several neuropsychiatric disorders. Effect Size Signed Differential Mapping [ES-SDM] (Radua et al. 2012) is a coordinate based metaanalytic technique that may be applied in VBM metaanalyses. ES-SDM accounts for within- and betweenstudy variance by combining peak coordinates and statistical parametric maps. As Radua and Mataix-Cols (2012) note, when comparing with regions of interest methods, voxel-based methods although comparatively less optimal in statistical terms, present the relevant advantage of a more exhaustive and unbiased inclusion of studies and brain regions.

The present meta-analysis aims to identify changes in cerebral morphometry and cognitive abilities in Alzheimer's disease by using the ES-SDM (Radua et al. 2012) and Open Meta-Analyst software (Wallace et al. 2009) packages. The identification of these changes will hopefully provide a detailed description of the PCA variant, further differentiating it from tAD.

\section{Methods}

\section{Data sources}

A comprehensive literature search was independently performed using PubMed, ScienceDirect and Web of Knowledge (see Online Table 1 for detailed search terms). No language or time restrictions were used in the search. Additionally, the bibliographies of relevant reviews and original articles were searched.

Studies selection

To be included in the meta-analysis, studies were required to have one PCA and one tAD group, which 
Table 1 Diagnostic criteria for typical AD and PCA - Summary of cardinal/defining features included in diagnostic criteria

\begin{tabular}{|c|c|c|c|c|}
\hline & $\begin{array}{l}\text { AD (based on } \\
\text { McKhann et al. 1984) }\end{array}$ & $\begin{array}{l}\text { AD-Amnestic presentation } \\
\text { (based on McKhann et al. 2011) }\end{array}$ & $\begin{array}{l}\text { AD-Visuospatial presentation } \\
\text { (Nonamnestic) (based on } \\
\text { McKhann et al. 2011) }\end{array}$ & $\begin{array}{l}\text { PCA (based on Kas et al. 2011; } \\
\text { and McMonagle et al. 2006) }\end{array}$ \\
\hline \multirow[t]{2}{*}{$\begin{array}{l}\text { Course and } \\
\text { presentation }\end{array}$} & $\begin{array}{l}\text { Progressive dementing } \\
\text { disorder manifesting } \\
\text { in middle or late life. }\end{array}$ & $\begin{array}{l}\text { Criteria for dementia should be } \\
\text { met with additional } \\
\text { characteristics of insidious } \\
\text { onset with clear-cut history of } \\
\text { worsening of cognition. }\end{array}$ & $\begin{array}{l}\text { Criteria for dementia should be } \\
\text { met with additional } \\
\text { characteristics of insidious onset } \\
\text { with clear-cut history of } \\
\text { worsening of cognition. }\end{array}$ & $\begin{array}{l}\text { Insidious onset and progressive } \\
\text { course of cognitive decline. }\end{array}$ \\
\hline & $\begin{array}{l}\text { Typically an insidious } \\
\text { onset of dementia } \\
\text { with progressive } \\
\text { memory and other } \\
\text { cognitive deficits. }\end{array}$ & $\begin{array}{l}\text { The initial and most prominent } \\
\text { cognitive deficits are in memory } \\
\text { (most common presentation of } \\
\text { AD). At least one other } \\
\text { cognitive domain is impaired. }\end{array}$ & $\begin{array}{l}\text { The initial and most prominent } \\
\text { cognitive deficits are in the } \\
\text { visuospatial domain (e.g. spatial } \\
\text { cognition, object agnosia, } \\
\text { impaired face recognition, } \\
\text { simultanagnosia, alexia). Other } \\
\text { cognitive domains should be } \\
\text { impaired. }\end{array}$ & $\begin{array}{l}\text { Marked visuospatial } \\
\text { dysfunction without } \\
\text { ophthalmologic impairment; } \\
\text { presence of Balint's and/or } \\
\text { Gerstmann's syndromes } \\
\text { symptoms. Proportionately } \\
\text { less memory loss or reduced } \\
\text { verbal fluency. }\end{array}$ \\
\hline \multirow[t]{3}{*}{ Considerations } & $\begin{array}{l}\text { Other psychiatric, } \\
\text { neurological or } \\
\text { medical disorders } \\
\text { should not account } \\
\text { for the observed } \\
\text { deficits. }\end{array}$ & \multicolumn{2}{|c|}{$\begin{array}{l}\text { Diagnosis of probable AD dementia should not be applied when there } \\
\text { is evidence of substantial concomitant cerebrovascular disease, core } \\
\text { features of other type of dementia, or if a medication or medical } \\
\text { condition influencing cognition are present. }\end{array}$} & $\begin{array}{l}\text { Focal neuroimaging lesions } \\
\text { (e.g. tumor, haematoma or } \\
\text { stroke) should be absent. }\end{array}$ \\
\hline & $\begin{array}{l}\text { The clinical criteria } \\
\text { include Possible, } \\
\text { Probable, and } \\
\text { Definite Alzheimer's } \\
\text { disease }\end{array}$ & \multicolumn{2}{|c|}{$\begin{array}{l}\text { Terminology includes: Probable AD; Possible AD dementia, and } \\
\text { Probable or possible AD dementia with evidence of the AD } \\
\text { pathophysiological process from (CSF or imaging) biomarkers. }\end{array}$} & \\
\hline & & \multicolumn{2}{|c|}{$\begin{array}{l}\text { Atypical course, and etiologically mixed presentations are also } \\
\text { considered. } \\
\text { The } 2011 \text { AD criteria revision includes non-amnestic presentations and } \\
\text { evidence of the AD pathophysiological process from (CSF or } \\
\text { imaging) biomarkers. }\end{array}$} & \\
\hline
\end{tabular}

ranged from early to late-onset, though, the presence of an HC group was not mandatory. Diagnoses of PCA and $\mathrm{tAD}$ had to be specifically mentioned in the study and performed according to established criteria (e.g. for PCA: Kas et al. 2011; McMonagle et al. 2006; for AD: McKhann et al. 1984; McKhann et al. 2011) (see Table 1 for a summary of diagnostic criteria). Studies reporting duplicate datasets were excluded. The present study follows the Preferred Reporting Items for Systematic Reviews and Meta-Analyses (PRISMA) guidelines (Moher et al. 2009).

Abstracts and full articles were independently reviewed for inclusion criteria by two of the authors (JA and JMS) with discrepancies being solved through discussion.

\section{Cognitive profiles}

Neuropsychological studies were included if they reported differential assessment of at least 1 PCA and 1 tAD (early or late-onset) group. Studies must also have provided the means and standard deviations of detailed neuropsychological assessments in at least 1 domain other than brief mental status tests or deterioration scales to be included. Studies returned by the neuroimaging literature search were also inspected for neuropsychological data. Full text articles or supplementary data were requested from authors when necessary. Studies that did not provide sufficient data for analysis were excluded.

Seven studies, which included a total of 104 PCA patients, $94 \mathrm{tAD}$ patients and $76 \mathrm{HC}$, were included in the present meta-analysis.

\section{Neuroimaging}

Inclusion criteria for neuroimaging studies were: 1) the existence of group comparisons using whole-brain VBM techniques, and 2) the presence of whole-brain group comparisons of gray and/or white matter volume or density via stereotactic coordinates corrected for multiple comparisons $(p<0.05)$. Four VBM studies, which reported whole-brain comparisons of gray matter for a total of 101 patients with PCA, 90 patients with tAD and $173 \mathrm{HC}$ were included in the final meta-analysis. 
Data extraction and data analysis

\section{Neuropsychological data}

OpenMeta-Analyst (Wallace et al. 2009) software was used to calculate effect sizes and conduct tests of heterogeneity for the included studies. Effect sizes were calculated using standardized between-group mean differences in cognitive performance and were measured using numerous neuropsychological instruments (PCA vs. tAD, PCA vs. HC, tAD vs. HC). Effect sizes were interpreted using Cohen's $d$ values $(0.2=$ small; $0.5=$ medium; $0.8=$ large $)$.

For each study included in our analysis, the number of participants per group, as well as the mean scores and standard deviations for each measure of cognitive ability were recorded. This was performed for all comparisons of neuropsychological test performance between patients with PCA and TAD, PCA patients and HC, and tAD patients and $\mathrm{HC}$, when available.

We required that a minimum of two studies assess a particular cognitive domain to consider that domain in our analysis (see Online Table 2 for the tests used in each study included by cognitive domain).

A detailed inspection of each study's findings was performed to integrate the neuropsychological data. The data were grouped into the following cognitive domains: Global Cognitive Functioning, Immediate Auditory/Verbal Memory, Delayed Auditory/Verbal Memory, Immediate Visuospatial Memory, Working Memory, Semantic Fluency, Phonemic Fluency, Language-Naming, Language-Comprehension, Visuoperceptive and Visuospatial Abilities, Ideomotor Praxis.

For each cognitive domain, pooled effect sizes were calculated using fixed-effects modeling or, in the case of substantial heterogeneity $\left(I^{2}>50 \%\right)$, random-effects (DerSimonian, and Laird 1986) modeling. Because fewer than ten studies were included for each meta-analysis, subgroup analysis, metaregression and analyses of publication bias, which use the Funnel Plot and Eggers' test, were not performed.
Neuroimaging data

The ES-SDM method (Radua et al. 2012) was used for the voxel-based meta-analysis. This method generates a coordinate map of GM differences, which are statistically significant at the whole-brain level and corrected for multiple comparisons, for each study. This map includes difference values that are both positive and negative; this prevents a single voxel to be significant in opposite directions. The mean of each study map, weighted by the square root of each study's respective sample size, was computed. This ensures that each study contributes proportionally to the resulting meta-analytic maps. In the preprocessing step, and in order to control for false-positives, a relatively wide full-width at half-maximum (FWHM, $20 \mathrm{~mm}$ ) was used and the statistical threshold was set to $p<0.005$ (Radua et al. 2012). To test the replicability of the results, a systematic whole-brain voxel-based jackknife sensitivity analysis was performed.

\section{Results}

Included studies

Four neuroimaging (Feldmann et al. 2008; Lehmann et al. 2011; Migliaccio et al. 2009; Whitwell et al. 2007) and eight neuropsychological (Aresi and Giovagnoli 2009; Caprile et al. 2009; Charles and Hillis 2005; Croisile et al. 2010; Kas et al. 2011; McMonagle et al. 2006; Migliaccio et al. 2009; Nestor et al. 2003;) studies met inclusion criteria (See Online Fig. 1 for the detailed flow diagram).

Five studies reported neuropsychological data for PCA, tAD and HC groups (see Online Table 2 for further details). All four neuroimaging studies reported data for these three groups as well. However, only two of the neuroimaging studies provided comparisons between PCA and tAD directly. The Croisile et al. (2010) study was not included in the

Table 2 Statistically significant effects results of PCA vs AD meta-analysis of cognitive domains

\begin{tabular}{|c|c|c|c|c|c|}
\hline Cognitive domain & $\begin{array}{l}\text { Studies } \\
(\mathrm{k})\end{array}$ & $\begin{array}{l}\text { Participants } \\
\text { (n) }\end{array}$ & Statistical method & $\begin{array}{l}\text { Estimated effect } \\
(95 \% \mathrm{CI})\end{array}$ & $\begin{array}{l}\text { Heterogeneity } \\
\text { Index }\left(I^{2}\right)\end{array}$ \\
\hline $\begin{array}{l}\text { Delayed auditory/verbal } \\
\text { memory }\end{array}$ & 4 & 102 & $\begin{array}{l}\text { Standardized Mean Difference (Fixed } \\
\text { Effects, } 95 \% \mathrm{CI} \text { ) }\end{array}$ & $\begin{array}{l}0.716(0.313,1.119) \\
p<0.001\end{array}$ & $0 \%$ \\
\hline Immediate visuospatial memory & 2 & 42 & $\begin{array}{l}\text { Standardized Mean Difference (Fixed } \\
\text { Effects, } 95 \% \mathrm{CI} \text { ) }\end{array}$ & $\begin{array}{l}-0.772(-1.399 \\
\quad-0.145) \\
p=0.016\end{array}$ & $0 \%$ \\
\hline $\begin{array}{l}\text { Visuoperceptive and } \\
\text { visuospatial abilities }\end{array}$ & 5 & 88 & $\begin{array}{l}\text { Standardized Mean Difference (Random } \\
\text { Effects, } 95 \% \text { CI) }\end{array}$ & $\begin{array}{l}-2.124(-3.546, \\
\quad-0.702) \\
p=0.003\end{array}$ & $83 \%$ \\
\hline
\end{tabular}

Negative effect sizes are interpreted as PCA group showing more impairment (higher test scores meaning best outcome) 
Fig. 1 Comparison of GM atrophy between PCA and AD groups. Blue: Greater atrophy in PCA vs AD; Red: Greater atrophy in AD compared to PCA. Neurologic view

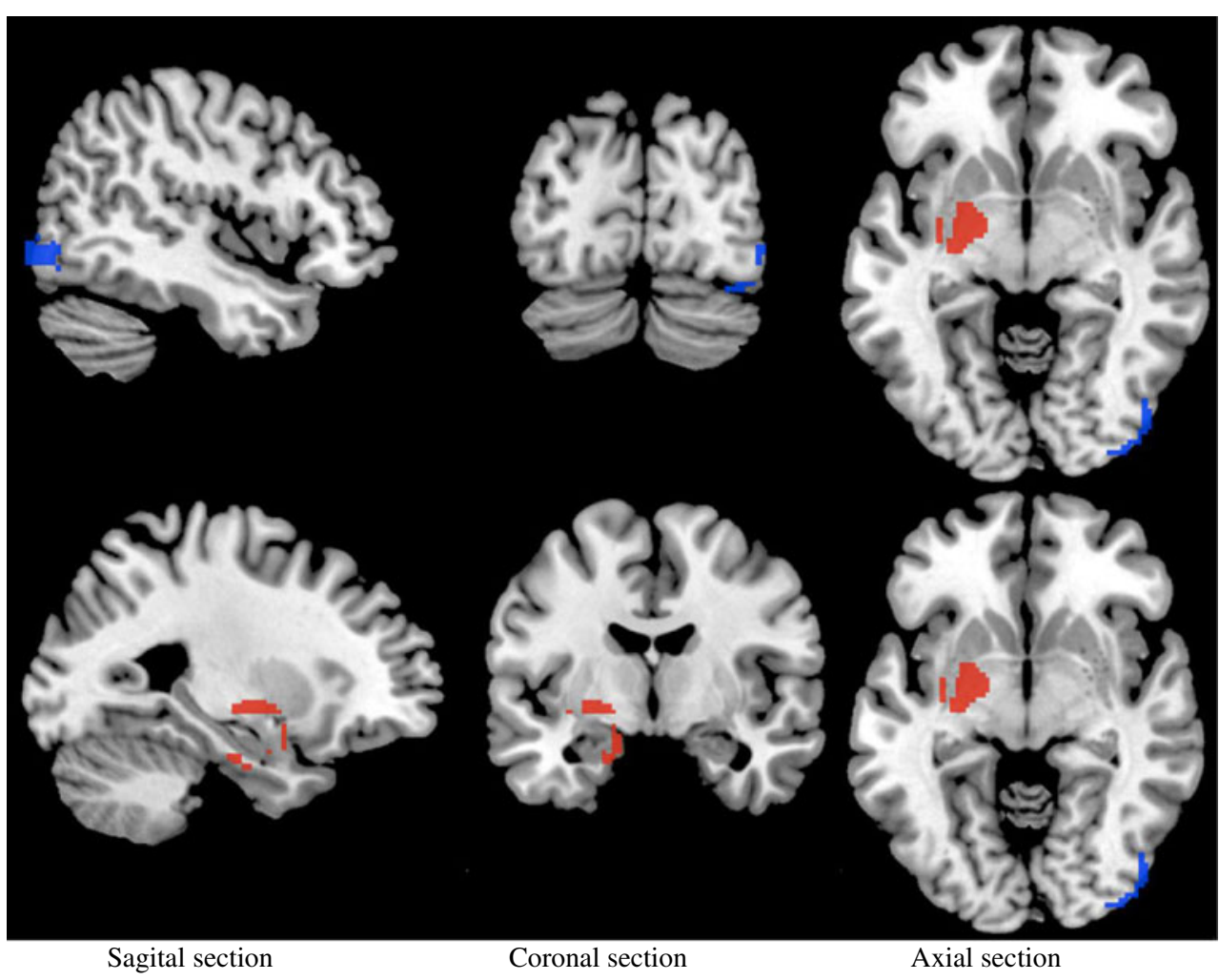

neuropsychological meta-analysis due to a large disparity in sample sizes for the mild-to-moderate Alzheimer's disease and PCA groups $(n=402, n=84$ vs. PCA $=16)$, resulting in seven neuropsychological studies (of eight meeting inclusion criteria) being included for the meta-analysis.

The studies included for analysis reported a mean MiniMental State Exam (MMSE) score greater than 14. The demographic and brief mental status data of the participants from all studies included in the analysis are presented in Online Table 3. The estimated mean difference of the MMSE yielded no significant differences in the amount of cognitive deterioration $(\mathrm{MD}=-0.820, p=0.226$ ) between the PCA and tAD groups. However, the VBM studies reported greater cognitive deterioration $(\mathrm{MD}=1.838, p=0.011)$ in the tAD group. One study (Whitwell et al. 2007) could not be included in this estimate because it did not provide mean values.

A total of 205 PCA patients, 184 tAD patients, and 249 $\mathrm{HC}$ participants were included in this study.

For each dataset, HC patients for comparison with PCA and tAD groups were the same for both groups, which would yield comparable global findings.

\section{Neuropsychological profile}

In most of the assessed domains, profiles of global cognitive impairments were observed in the PCA and tAD patient groups (see Online Tables 4 and 5 and 6 for detailed results). Compared to tAD patients, PCA patients exhibited significantly greater impairment in Immediate Visuospatial
Memory (Standardized mean difference $[\mathrm{SMD}]=-0.772$, $\left.p=0.016, I^{2}=0 \%\right)$, as well as Visuoperceptive and Visuospatial Abilities (SMD $=-2.124, p=0.003, I^{2}=83 \%$ ).

However, tAD patients showed significantly greater impairment in Delayed Auditory/Verbal Memory than patients with PCA $\left(\mathrm{SMD}=0.716, p<0.001, I^{2}=0 \%\right.$; see Table 2 for statistically significant results; see Online Table 6 for detailed results).

\section{Brain morphometry (Gray matter VBM meta-analysis: whole-brain gray matter differences between $t A D, P C A$ and $\mathrm{HC}$ )}

Only studies with VBM volumetric assessment were found in the literature (i.e. no studies using other segmentation methods - e.g., FreeSurfer, FSL-were found).

Studies included in the meta-analysis indicated reductions of gray matter volume in the middle and superior temporal gyri, the fusiform gyrus extending to the left caudate tail and in the left parahippocampal gyrus extending to the globus pallidus and putamen, which was significantly greater for tAD patients than for HC participants. Compared to $\mathrm{HC}$, PCA patients exhibited significantly greater gray matter reductions in the inferior and medial left temporal gyri extending to the occipital gyrus. Gray matter volume reductions were also observed in PCA patients in the left fusiform and parahippocampal gyri as well as the right parietal lobule, including the inferior middle and superior parietal and angular gyri. However, when compared to tAD 
Table 3 Gray matter differences between PCA, AD and HC

\begin{tabular}{|c|c|c|c|c|c|c|c|}
\hline \multirow[t]{2}{*}{ Comparison } & \multirow[t]{2}{*}{ Region } & \multicolumn{3}{|l|}{ Maximum } & \multicolumn{2}{|l|}{ Cluster } & \multirow{2}{*}{$\begin{array}{l}\text { Jackknife } \\
\text { sensitivity analysis }\end{array}$} \\
\hline & & $\begin{array}{l}\text { Talairach } \\
\text { coordinates x y z }\end{array}$ & $\begin{array}{l}\text { SDM } \\
\text { value }\end{array}$ & $P$ value & $\begin{array}{l}\text { Number of } \\
\text { voxels }\end{array}$ & Breakdown (number of voxels) & \\
\hline \multirow{8}{*}{$\begin{array}{l}\text { AD vs } \\
\text { Controls }\end{array}$} & \multirow[t]{5}{*}{ Left temporal gyrus } & \multirow[t]{5}{*}{$-34-26-6$} & \multirow[t]{5}{*}{-2.762} & \multirow[t]{5}{*}{0.00002} & \multirow[t]{5}{*}{358} & Left caudate, caudate tail (12) & 3 out of 4 \\
\hline & & & & & & Left sub-gyral hippocampus (34) & 3 out of 4 \\
\hline & & & & & & Left middle temporal gyrus (63) & 3 out of 4 \\
\hline & & & & & & Left superior temporal gyrus (76) & 3 out of 4 \\
\hline & & & & & & Left fusiform gyrus (52) & 3 out of 4 \\
\hline & \multirow{3}{*}{$\begin{array}{l}\text { Left } \\
\text { parahippocampal } \\
\text { gyrus }\end{array}$} & \multirow[t]{3}{*}{$-16-6-16$} & \multirow[t]{3}{*}{-1.679} & \multirow[t]{3}{*}{0.001} & \multirow[t]{3}{*}{292} & Left parahippocampal gyrus (86) & 4 out of 4 \\
\hline & & & & & & $\begin{array}{l}\text { Left lentiform nucleus, medial } \\
\text { globus pallidus }(51)\end{array}$ & 2 out of 4 \\
\hline & & & & & & $\begin{array}{l}\text { Left lentiform nucleus, putamen } \\
\text { (15) }\end{array}$ & 3 out of 4 \\
\hline \multirow{9}{*}{$\begin{array}{l}\text { PCA vs } \\
\text { Controls }\end{array}$} & \multirow{3}{*}{$\begin{array}{l}\text { Left inferior } \\
\text { temporal gyrus }\end{array}$} & \multirow[t]{3}{*}{$-46-680$} & \multirow[t]{3}{*}{-2.199} & \multirow[t]{3}{*}{0.0001} & \multirow[t]{3}{*}{80} & Left inferior temporal gyrus (22) & 3 out of 4 \\
\hline & & & & & & Left middle temporal gyrus (29) & 3 out of 4 \\
\hline & & & & & & Left middle occipital gyrus (19) & 3 out of 4 \\
\hline & \multirow[t]{2}{*}{ Left fusiform gyrus } & \multirow[t]{2}{*}{$-28-48-10$} & \multirow[t]{2}{*}{-2.186} & \multirow[t]{2}{*}{0.0001} & \multirow[t]{2}{*}{204} & Left fusiform gyrus (39) & 3 out of 4 \\
\hline & & & & & & Left parahippocampal gyrus (45) & 3 out of 4 \\
\hline & \multirow{4}{*}{$\begin{array}{l}\text { Right inferior } \\
\text { parietal lobule }\end{array}$} & \multirow[t]{4}{*}{$42-4638$} & \multirow[t]{4}{*}{-1.870} & \multirow[t]{4}{*}{0.0007} & \multirow[t]{4}{*}{457} & Right inferior parietal lobule (168) & 2 out of 4 \\
\hline & & & & & & Right middle temporal gyrus (111) & 2 out of 4 \\
\hline & & & & & & Right superior temporal gyrus (57) & 2 out of 4 \\
\hline & & & & & & Right angular gyrus (66) & 2 out of 4 \\
\hline \multirow[t]{5}{*}{ PCA vs AD } & Right middle & $48-78-6$ & -1.007 & 0.0003 & 173 & Right middle occipital gyrus (16) & \\
\hline & occipital gyrus & & & & & Right inferior occipital gyrus (91) & \\
\hline & & & & & & Right posterior lobe (57) & \\
\hline & Left & $-34-18-12$ & 1.007 & 0.0004 & 318 & Left parahippocampal gyrus, hippoc & campus (24) \\
\hline & $\begin{array}{l}\text { parahippocampal } \\
\text { gyrus }^{\mathrm{a}}\end{array}$ & & & & & Left parahippocampal gyrus (121) & \\
\hline
\end{tabular}

${ }^{\mathrm{a}}$ Increased gray matter

patients, PCA patients showed significant decreases in gray matter volume in the middle and inferior right occipital gyri, extending to the posterior lobule. PCA patients also exhibited greater volume sparing in the left parahippocampal gyrus and the left hippocampus (Fig. 1 illustrates the results from the comparison between the PCA and tAD groups; for a comparison between PCA, tAD and $\mathrm{HC}$ groups, see Online Fig. 2).

The replicability of these results was assessed using a systematic whole-brain voxel-based jackknife sensitivity analysis, which was based on the exclusion of one study per iteration. As presented in Table 3, this analysis showed that the main results of this study were replicable.

\section{Discussion}

To the best of our knowledge this is the first metaanalysis providing a characterization of both neuropsychological and brain morphometric features of PCA when compared with typical tAD and HC.
Compared to HC, PCA patients showed atrophy of brain areas similar to tAD patients. In particular, volume loss was observed in the left fusiform, left parahippocampal gyrus and left middle temporal gyrus, which represents a shared pattern of wide temporal atrophy. PCA patients exhibited significantly more atrophy in the right occipital gyrus and right posterior lobe than tAD patients, whereas atrophy in the left parahippocampal gyrus and hippocampus was greater in the tAD group. Moreover, these neuroimaging data were consistent with findings from the neurocognitive literature; individuals with PCA showed higher levels of impairment in Visuoperceptive and Visuospatial Abilities and Immediate Visuospatial Memory. In addition, tAD patients presented with greater impairments in Delayed Auditory/Verbal Memory. These findings support an association between neural atrophy and the cognitive deficits observed in PCA and tAD.

PCA and tAD probably result from the same neurodegenerative process. While PCA and tAD share some regions of brain atrophy, the patterns of atrophy and neuropsychological characteristics of these conditions 
may differ. Both of these variants can be distinguished by structural neuroimaging and neuropsychological assessment.

The findings of this study support previous neuropsychological studies (Galton et al. 2000) and reviews (Crutch et al. 2012), which show that compared to tAD, the mild-tomoderate stages of PCA are characterized by severe deficits in visuospatial abilities, which distinguish it from typical tAD (where there is comparably more impairment in Delayed Auditory/Verbal Memory). These data corroborate findings from neuroimaging studies, which localize brain atrophy to predominantly posterior areas (Crutch et al. 2012). Specifically, atrophy of occipital and parietal regions may result in the visuospatial deficits that have been previously described in PCA (Crutch et al. 2012). In addition, functional neuroimaging studies have shown a pattern of hypometabolism and functional underactivation (Crutch et al. 2012; Feldmann et al. 2008) in posterior areas (the parietal and/or occipital lobes). This could potentially be related to the increased deposition of senile plaques in these areas observed in PCA (Crutch et al. 2012).

In the present study, PCA patients exhibited more marked volume reduction than that for tAD patients in the right occipital gyrus (middle, inferior and posterior occipital lobe). The mechanisms underlying this pattern of atrophy remain unclear.

In tAD patients, extensive atrophy was observed in the medial temporal lobe, in particular, the left hippocampal region, which has previously been associated with impairments in encoding and retrieval of episodic memory (Yamasaki et al. 2012). Although brain atrophy was limited to the temporal, parietal and occipital lobes of PCA patients, we observed a global pattern of cognitive impairment when compared to HC (e.g., impairment in Semantic and Phonemic Fluency). Besides indicating memory impairments, these fluency deficits are also indicative of executive impairments. While no atrophy was found in the frontal lobes of PCA patients, it is possible that functional underactivation of this area may underlie these deficits and precede structural abnormalities. Functional impairments, such as hypometabolism, may be present during earlier stages of the disease (Nestor et al. 2003). They may eventually progress to frank structural damage, possibly beginning in the milder stages of the disease or later.

Another intriguing cognitive aspect is the relationship between visual memory and visuoperceptive and visuospatial abilities. While the possibility of visuoperceptive deficits originating the marked pattern of visual memory dysfunction in PCA patients seems viable, this remains a plausible hypothesis to be tested. In the present study, PCA patients showed more marked deficits in immediate visuospatial memory, visuoperceptive and visuospatial abilities than $\mathrm{AAD}$; while $\mathrm{AD}$ patients showed marked deficits in
Immediate Auditory/Verbal Memory, when compared with PCA. Unfortunately, it was not possible to perform an analysis regarding the delayed visuospatial memory domain in PCA patients due to the lack of a minimum of 2 studies with compatible cognitive tests for this domain. Future research, extensively characterizing the natural course of visual memory deficits and each cognitive function might clarify this issue.

Additionally, and consistent with previous studies, we found atrophy in the globus pallidus and putamen. For example, Josephs et al. (2006) reported evidence of atrophy in globus pallidus that may be associated with visual hallucinations. Whitwell et al. (2007) found also evidence for extended atrophy in regions spreading from the parietal lobes to primary sensory and motor cortex. However, the full clinical meaning of atrophy in these regions remains to be clarified.

GM atrophy may progress from a focal to a global presentation, which is not confined to areas associated with the initial symptomatology (Lehmann et al. 2012) of PCA and tAD. This may be congruent with the spread of pathology observed over time in these conditions as well.

Morphometric changes can also occur in the WM. For example, PCA reduces the integrity of WM tracts in posterior brain regions. This may result in network-level dysfunctions affecting the major visual pathways predominantly in the right hemisphere (Crutch et al. 2012; Migliaccio et al. 2012a, b).

Some limitations of the present study should be acknowledged. First, patients sampled in the studies used for this analysis differed in the stage and/or severity of the disease, as evidenced by different MMSE scores. In addition, only a limited number of studies were appropriate for analysis, which is especially true for the neuroimaging literature, which discourages performing sub-group analysis or metaregression. Therefore, it was not possible to perform a thorough assessment of the impact of variables such as age, gender, education and MMSE in the observed results. Similarly, the relation between specific domains (e.g. immediate visual memory and visuoperceptive and visuospatial abilities) and the characterization of some specific cognitive functions (e.g. delayed visuospatial memory) could not be currently established. Because the frequency of PCA presentation is relatively low, PCA is likely to be under recognized and understudied. Moreover, the PCA entity has some circularity since the diagnostic criteria used for PCA demands visuospatial dysfunction in excess of episodic memory dysfunction, with the name of the diagnostic entity predicting the regionally specific atrophy pattern. We hope that our analysis of the neuropsychological and imaging data will improve the diagnostic and clinical understandings of PCA and contribute to the nosological and etiological characterization of each type of dementia presentation 
through the inclusion of detailed quantitative neuroimaging and neuropsychological PCA profiles in diagnostic criteria. In this regard, we believe that the standardization of cognitive and neuroimaging parameters (e.g. obtaining group mean values for each cognitive domain and neuroimaging parameter-e.g. volumetry - throughout the course of the disease) for each $\mathrm{AD}$ variant and other dementias might be an important aspect allowing to empirically define the PCA profile and other variants of AD. This way it would be possible to quantify how much a set of cognitive functions/domains and structural and functional neuroimaging parameters (e.g. hippocampal, parietal, occipital volume, etc.) deviate from a set of normative values. These comparisons could be performed either at the inter-dementia class or within each dementia (e.g. between $\mathrm{AD}$ variants). For example, a clinician might be able to generate memory and hippocampal volume z-scores for the patient with suspected PCA and compare it with a set of standardized values of healthy subjects, $\mathrm{AD}$ and other dementia patients. Similarly, the same comparison would be generated for other cognitive domains and structures allowing to choose the more probable diagnosis based on intra and interdementia comparisons.

Further longitudinal follow-up studies are necessary to understand the natural progression of PCA, as well as its different neural (atrophy, abnormal fMRI/PET brain activation patterns) and cognitive markers that characterize the stages of PCA. Once the imaging correlations and changes in cognitive profiles in PCA are more fully described, diagnostic accuracy and clinical care of the disorder may be improved.

Reliable markers to diagnose PCA are presented in our analysis. Because cognitive testing and structural MRI may be more readily available and less expensive than other imaging techniques (e.g. PET imaging), we believe that using these markers may be potentially efficacious in the diagnosis of PCA. Moreover structural changes such as synaptic/neuronal loss correlate better than plaques or tangles with cognitive deficits (Shankar and Walsh 2009; Terry et al. 1991). Structural pathology databases may one day be worldwide available in clinical settings to make automatic comparisons of brain volume loss, which could assist in the identification of PCA.

In summary, PCA is characterized by a global profile of cognitive impairment, marked by visuospatial deficits. In particular, PCA can be distinguished from tAD by GM changes and marked deficits in Visuoperceptive and Visuospatial Abilities and Immediate Visuospatial Memory. Additionally, tAD is characterized by impairments in Delayed Auditory/Verbal Memory, as well as temporal lobe atrophy. As Crutch et al. (2012) noted, PCA is a focal degenerative syndrome, which lacks consistent and standardized diagnostic criteria. In this study we provided a systematic review of the neural and cognitive correlates of PCA. We also demonstrated that global cognitive impairments are observed in this condition, the most severe of which is visuospatial dysfunction.

The findings of this study could help to elucidate the current debate surrounding the classification of PCA as Alzheimer's disease visual variant and allow for a better understanding and awareness of PCA and a more standardized diagnosis. We hope this work may provide working hypotheses and promote new research in this field, since the current limited amount of data precludes the establishment of well-founded conclusions, which would advance diagnostic and treatment guidelines.

Acknowledgments JA is funded by a doctoral grant from the Foundation for Science and Technology, FCT (SFRH/BD/64457/2009, cofunded by FSE/POPH). JA and AS are funded by project PIC/IC/ $83290 / 2007$, which is supported by FEDER (POFC - COMPETE) and FCT. JMS is supported by a fellowship of the project SwitchBox-FP7-HEALTH-2010-grant 259772-2. These organizations had no role in the study design, data collection, analysis, interpretation, or in the decision to submit the paper for publication.

Conflict of interest The authors declare that they have no conflict of interest.

\section{References}

Amieva, H., Phillips, L. H., Della Sala, S., \& Henry, J. D. (2004). Inhibitory functioning in Alzheimer's disease. Brain, 127(5), 949-964. doi:10.1093/brain/awh045.

Aresi, A., \& Giovagnoli, A. R. (2009). The role of neuropsychology in distinguishing the posterior cortical atrophy syndrome and Alzheimer's disease. Journal of Alzheimer's Disease, 18(1), 6570. doi:10.3233/JAD-2009-1123.

Ashburner, J., \& Friston, K. J. (2000). Voxel-based morphometry-the methods. Neuroimage, 11(6), 805-821. doi:10.1006/nimg.2000.0582.

Backman, L., Jones, S., Berger, A. K., Laukka, E. J., \& Small, B. J. (2004). Multiple cognitive deficits during the transition to Alzheimer's disease. Journal of Internal Medicine, 256(3), 195204. doi:10.1111/j.1365-2796.2004.01386.x.

Benson, F., Davis, J., \& Snyder, B. D. (1988). Posterior cortical atrophy. Archives of Neurology, 45, 789-793.

Braak, H., \& Braak, E. (1991). Neuropathological stageing of Alzheimer-related changes. Acta Neuropathologica, 82(4), 239259.

Caprile, C., Bosch, B., Rami, L., Sanchez-Valle Diaz, R., Bartres-Faz, D., \& Molinuevo, J. L. (2009). Posterior cortical atrophy. Its neuropsychological profile and differences from typical Alzheimer's disease. Revista de Neurologia, 48(4), 178-182.

Charles, R. F., \& Hillis, A. E. (2005). Posterior cortical atrophy: clinical presentation and cognitive deficits compared to Alzheimer's disease. Behavioural Neurology, 16(1), 15-23.

Clare, L., Woods, R. T., Moniz Cook, E. D., Orrell, M., \& Spector, A. (2003). Cognitive rehabilitation and cognitive training for earlystage Alzheimer's disease and vascular dementia. Cochrane Database of Systematic Reviews (4), CD003260. doi:10.1002/ 14651858.CD003260

Croisile, B. (2004). Benson's syndrome or Posterior Cortical Atrophy. Orphanet Encyclopedia. http://www.orpha.net/data/patho/GB/ukBenson.pdf. Accessed 30 September 2012. 
Croisile, B., Astier, J. L., Beaumont, C., \& Mollion, H. (2010). Validation of the Rapid BAttery of Denomination (BARD) in 382 controls and 1004 patients of a memory clinic. Revue Neurologique (Paris), 166(6-7), 584-593. doi:10.1016/j.neurol.2010.01.017.

Crutch, S. J., Lehmann, M., Schott, J. M., Rabinovici, G. D., Rossor, M. N., \& Fox, N. C. (2012). Posterior cortical atrophy. Lancet Neurology, 11(2), 170-178. doi:10.1016/S1474-4422(11)70289-7.

de Souza, L. C., Corlier, F., Habert, M. O., Uspenskaya, O., Maroy, R., Lamari, F., et al. (2011). Similar amyloid-beta burden in posterior cortical atrophy and Alzheimer's disease. Brain, 134(7), 2036 2043. doi:10.1093/brain/awr130.

DerSimonian, R., \& Laird, N. (1986). Meta-analysis in clinical trials. Controlled Clinical Trials, 7(3), 177-188. doi:10.1016/01972456(86)90046-2.

Feldmann, A., Trauninger, A., Toth, L., Kotek, G., Kosztolanyi, P., Illes, E., et al. (2008). Atrophy and decreased activation of frontoparietal attention areas contribute to higher visual dysfunction in posterior cortical atrophy. Psychiatry Research, 164(2), 178-184. doi:10.1016/j.pscychresns.2008.01.006.

Formaglio, M., Costes, N., Seguin, J., Tholance, Y., Le Bars, D., Roullet-Solignac, I., et al. (2011). In vivo demonstration of amyloid burden in posterior cortical atrophy: a case series with PET and CSF findings. Journal of Neurology, 258(10), 1841-1851. doi:10.1007/s00415-011-6030-0.

Galton, C. J., Patterson, K., Xuereb, J. H., \& Hodges, J. R. (2000). Atypical and typical presentations of Alzheimer's disease: a clinical, neuropsychological, neuroimaging and pathological study of 13 cases. Brain, 123(3), 484-498.

Josephs, K. A., Whitwell, J. L., Boeve, B. F., Knopman, D. S., TangWai, D. F., Drubach, D. A., et al. (2006). Visual hallucinations in posterior cortical atrophy. Archives of Neurology, 63(10), 14271432. doi:10.1001/archneur.63.10.1427.

Kas, A., de Souza, L. C., Samri, D., Bartolomeo, P., Lacomblez, L., Kalafat, M., et al. (2011). Neural correlates of cognitive impairment in posterior cortical atrophy. Brain, 134(5), 1464-1478. doi:10.1093/brain/awr055.

Lehmann, M., Crutch, S. J., Ridgway, G. R., Ridha, B. H., Barnes, J., Warrington, E. K., et al. (2011). Cortical thickness and voxelbased morphometry in posterior cortical atrophy and typical Alzheimer's disease. Neurobiology of Aging, 32(8), 1466-1476. doi:10.1016/j.neurobiolaging.2009.08.017.

Lehmann, M., Barnes, J., Ridgway, G. R., Ryan, N. S., Warrington, E. K., Crutch, S. J., et al. (2012). Global gray matter changes in posterior cortical atrophy: a serial imaging study. Alzheimer's \& Dementia, 8(6), 502-512. doi:10.1016/j.jalz.2011.09.225.

McKhann, G., Drachman, D., Folstein, M., Katzman, R., Price, D., \& Stadlan, E. M. (1984). Clinical diagnosis of Alzheimer's disease: report of the NINCDS-ADRDA Work Group under the auspices of Department of Health and Human Services Task Force on Alzheimer's Disease. Neurology, 34(7), 939-944.

McKhann, G. M., Knopman, D. S., Chertkow, H., Hyman, B. T., Jack, C. R., Jr., Kawas, C. H., et al. (2011). The diagnosis of dementia due to Alzheimer's disease: recommendations from the National Institute on Aging-Alzheimer's Association workgroups on diagnostic guidelines for Alzheimer's disease. Alzheimer's \& Dementia, 7(3), 263-269. doi:10.1016/j.jalz.2011.03.005.

McMonagle, P., Deering, F., Berliner, Y., \& Kertesz, A. (2006). The cognitive profile of posterior cortical atrophy. Neurology, 66(3), 331-338. doi:10.1212/01.wnl.0000196477.78548.db.

Migliaccio, R., Agosta, F., Rascovsky, K., Karydas, A., Bonasera, S., Rabinovici, G. D., et al. (2009). Clinical syndromes associated with posterior atrophy: early age at onset AD spectrum. Neurology, 73(19), 1571-1578. doi:10.1212/WNL.0b013e3181c0d427.

Migliaccio, R., Agosta, F., Possin, K. L., Rabinovici, G. D., Miller, B. L., \& Gorno-Tempini, M. L. (2012a). White matter atrophy in Alzheimer's disease variants. Alzheimer's \& Dementia, $8(5$ Suppl), S78-S87. doi:10.1016/j.jalz.2012.04.010. e71-72.

Migliaccio, R., Agosta, F., Toba, M. N., Samri, D., Corlier, F., de Souza, L. C., et al. (2012b). Brain networks in posterior cortical atrophy: a single case tractography study and literature review. Cortex, 48(10), 1298-1309. doi:10.1016/j.cortex.2011.10.002.

Moher, D., Liberati, A., Tetzlaff, J., \& Altman, D. G. (2009). Preferred reporting items for systematic reviews and meta-analyses: the PRISMA statement. PLoS Medicine, 6(7), e1000097. doi:10.1371/journal.pmed.1000097.

Nestor, P. J., Caine, D., Fryer, T. D., Clarke, J., \& Hodges, J. R. (2003). The topography of metabolic deficits in posterior cortical atrophy (the visual variant of Alzheimer's disease) with FDG-PET. Journal of Neurology, Neurosurgery \& Psychiatry, 74(11), 1521-1529. doi:10.1136/jnnp.74.11.1521.

Radua, J., \& Mataix-Cols, D. (2012). Meta-analytic methods for neuroimaging data explained. Biology of Mood \& Anxiety Disorders, 2(1), 6. doi:10.1186/2045-5380-2-6.

Radua, J., Mataix-Cols, D., Phillips, M. L., El-Hage, W., Kronhaus, D. M., Cardoner, N., et al. (2012). A new meta-analytic method for neuroimaging studies that combines reported peak coordinates and statistical parametric maps. Eur Psychiatry, 27(8), 605-611. doi:10.1016/j.eurpsy.2011.04.001.

Scahill, R. I., Schott, J. M., Stevens, J. M., Rossor, M. N., \& Fox, N. C. (2002). Mapping the evolution of regional atrophy in Alzheimer's disease: unbiased analysis of fluid-registered serial MRI. Proceedings of the National Academy of Sciences of the United States of America, 99(7), 4703-4707. doi:10.1073/pnas.052587399.

Shankar, G. M., \& Walsh, D. M. (2009). Alzheimer's disease: synaptic dysfunction and Abeta. Molecular Neurodegeneration, 4, 48. doi:10.1186/1750-1326-4-48.

Terry, R. D., Masliah, E., Salmon, D. P., Butters, N., DeTeresa, R., Hill, R., et al. (1991). Physical basis of cognitive alterations in Alzheimer's disease: synapse loss is the major correlate of cognitive impairment. Annals of Neurology, 30(4), 572-580. doi:10.1002/ana.410300410.

Victoroff, J., Ross, G. W., Benson, D. F., Verity, M. A., \& Vinters, H. V. (1994). Posterior cortical atrophy. Neuropathologic correlations. Archives of Neurology, 51(3), 269-274.

Wallace, B. C., Schmid, C. H., Lau, J., \& Trikalinos, T. A. (2009). Meta-Analyst: software for meta-analysis of binary, continuous and diagnostic data. BMC Medical Research Methodology, 9, 80. doi:10.1186/1471-2288-9-80.

Westman, E., Cavallin, L., Muehlboeck, J. S., Zhang, Y., Mecocci, P., Vellas, B., et al. (2011). Sensitivity and specificity of medial temporal lobe visual ratings and multivariate regional MRI classification in Alzheimer's disease. PLoS One, 6(7), e22506. doi:10.1371/journal.pone.0022506.

Whitwell, J. L., Jack, C. R., Jr., Kantarci, K., Weigand, S. D., Boeve, B. F., Knopman, D. S., et al. (2007). Imaging correlates of posterior cortical atrophy. Neurobiology of Aging, 28(7), 10511061. doi:10.1016/j.neurobiolaging.2006.05.026.

Yamasaki, T., Muranaka, H., Kaseda, Y., Mimori, Y., \& Tobimatsu, S. (2012). Understanding the pathophysiology of Alzheimer's disease and mild cognitive impairment: a mini review on fMRI and ERP studies. Neurology Research International, 2012, 719056. doi:10.1155/2012/719056. 